\title{
The radius and effective temperature of the binary Ap star $\beta$ CrB from CHARA/FLUOR and VLT/NACO observations ${ }^{\star}$
}

\author{
H. Bruntt ${ }^{1,2}$, P. Kervella ${ }^{1}$, A. Mérand ${ }^{3}$, I. M. Brandão ${ }^{4,5}$, T. R. Bedding ${ }^{2}$, T. A. ten Brummelaar ${ }^{6}$, V. Coudé du Foresto ${ }^{1}$, \\ M. S. Cunha ${ }^{4}$, C. Farrington ${ }^{6}$, P. J. Goldfinger ${ }^{6}$, L. L. Kiss ${ }^{2,7}$, H. A. McAlister ${ }^{6}$, S. T. Ridgway ${ }^{8}$, J. Sturmann ${ }^{6}$, \\ L. Sturmann ${ }^{6}$, N. Turner ${ }^{6}$, and P. G. Tuthill ${ }^{2}$
}

${ }^{1}$ LESIA, CNRS UMR 8109, Observatoire de Paris-Meudon, 5 place Jules Janssen, 92195 Meudon Cedex, France e-mail: bruntt@phys.au.dk

2 Sydney Institute for Astronomy, School of Physics, The University of Sydney, NSW 2006, Australia

3 European Southern Observatory, Alonso de Córdova 3107, Casilla 19001, Santiago 19, Chile

4 Universidade do Porto, Centro de Astrofísica, Rua das Estrelas, 4150-762 Porto, Portugal

5 Departamento de Matemática Aplicada, Faculdade de Ciências, Universidade do Porto, 4169 Porto, Portugal

6 Center for High Angular Resolution Astronomy, Georgia State University, PO Box 3965, Atlanta, Georgia 30302-3965, USA

7 Konkoly Observatory of the Hungarian Academy of Sciences, Budapest, Hungary

8 National Optical Astronomy Observatory, PO box 26732, Tucson, AZ 85726, USA

Received 5 October 2009 / Accepted 10 December 2009

\begin{abstract}
Context. The prospects for using the asteroseismology of rapidly oscillating Ap (roAp) stars are hampered by the large uncertainty in fundamental stellar parameters. Results in the literature for the effective temperature $\left(T_{\text {eff }}\right)$ often span a range of $1000 \mathrm{~K}$.

Aims. Our goal is to reduce systematic errors and improve the $T_{\text {eff }}$ calibration of Ap stars based on new interferometric measurements. Methods. We obtained long-baseline interferometric observations of $\beta \mathrm{CrB}$ using the CHARA/FLUOR instrument. To disentangle the flux contributions of the two components of this binary star, we obtained VLT/NACO adaptive optics images.

Results. We determined limb-darkened angular diameters of $0.699 \pm 0.017$ mas for $\beta \mathrm{CrB}$ A (from interferometry) and $0.415 \pm$ 0.017 mas for $\beta \mathrm{CrB} \mathrm{B}$ (from surface brightness-colour relations), corresponding to radii of $2.63 \pm 0.09 R_{\odot}$ (3.4\% uncertainty) and $1.56 \pm 0.07 R_{\odot}(4.5 \%)$. The combined bolometric flux of the $\mathrm{A}+\mathrm{B}$ components was determined from satellite UV data, spectrophotometry in the visible, and broadband data in the infrared. The flux from the B component constitutes $16 \pm 4 \%$ of the total flux and was determined by fitting an ATLAS9 model atmosphere to the broad-band NACO $J$ and $K$ magnitudes. By combining the flux of the A component with its measured angular diameter, we determined the effective temperature $T_{\text {eff }}(\mathrm{A})=7980 \pm 180 \mathrm{~K}(2.3 \%)$. Conclusions. Our new interferometric and imaging data enable nearly model-independent determination of the effective temperature of $\beta \mathrm{CrB}$ A. Including our recent study of $\alpha \mathrm{Cir}$, we now have direct $T_{\text {eff }}$ measurements of two of the brightest roAp stars, providing a strong benchmark for improved calibration of the $T_{\text {eff }}$ scale for Ap stars. This will support the use of potentially strong constraints imposed by asteroseismic studies of roAp stars.
\end{abstract}

Key words. stars: chemically peculiar - stars: fundamental parameters - stars: individual: $\beta \mathrm{CrB}$ - stars: individual: $\alpha$ Cir stars: individual: $\gamma$ Equ - stars: individual: $10 \mathrm{Aql}$

\section{Introduction}

Photometric and spectroscopic determinations of the effective temperatures of Ap stars are affected by systematic errors. This has been corroborated by the asteroseismic data of rapidly oscillating Ap (roAp) stars in general and, more recently, by the first interferometric determination of the angular diameter of the roAp star $\alpha$ Cir (Bruntt et al. 2008). Unfortunately, the intriguing asteroseismic potential offered by roAp stars is strongly compromised by these systematic errors. We therefore seek to directly measurement of the radii and effective temperatures of a number of Ap stars using interferometry and spectrophotometry. We first give a brief summary of the properties of $\beta \mathrm{CrB}$ before describing our observations, data reduction (Sect. 2), and analysis (Sect. 3).

$\beta \mathrm{CrB}$ (HD 137909, HIP 75695) is one of the brightest, coolest, and best-studied magnetic Ap stars. The literature on

^ Based on observations made with ESO telescopes at the La Silla Paranal Observatory, under ESO DDT programme 281.D-5020(A). the star is extensive and we only mention a few of the most important results here. It was classified as a type $\mathrm{A} 9 \mathrm{SrEu} \mathrm{Cr}$ star by Renson \& Manfroid (2009). Its binary nature was first suggested by Campbell \& Moore (1907), and recent determinations of its orbital elements have been obtained by Tokovinin (1984) and North et al. (1998). From speckle interferometric measurements using narrow-band filters, Horch et al. (2004) measured the magnitude difference to be $2.37 \mathrm{mag}$ at $551 \mathrm{~nm}$ and $1.99 \mathrm{mag}$ at $503 \mathrm{~nm}$. In the analysis presented in Sect. 3 we retain the orbital elements obtained by Tokovinin (1984), as they are in significantly better agreement with our NACO astrometry than those by North et al. (1998).

Neubauer (1944) suggested that a third body could be present in the system, causing radial velocity variations with a period of $\approx 321$ days, but Oetken \& Orwert (1984), Kamper et al. (1990), and Söderhjelm (1999) excluded this possibility. Recently, Muterspaugh et al. (2006) established an upper limit of $\approx 10$ to $100 M_{J}$ (depending on the orbital period) for a possible substellar tertiary from differential interferometric astrometry. 
Trilling et al. (2007) searched for 24 and $70 \mu \mathrm{m}$ infrared excess around $\beta \mathrm{CrB}$ using Spitzer but did not find any. Interestingly, the Spitzer flux they obtained is significantly below the expected flux at $24 \mu \mathrm{m}$, and slightly lower (although compatible) at $70 \mu \mathrm{m}$. This result could come from the chosen physical parameters for their stellar atmosphere model. In the following, we will therefore consider that $\beta \mathrm{CrB}$ is a binary system.

Early photometric searches for pulsation in $\beta \mathrm{CrB}$ (e.g. Weiss $\&$ Schneider 1989; Kreidl 1991) gave null results and this contributed to the discussion of the existence of non-oscillating Ap stars ("noAp"; Kurtz 1989). This has changed since the advent of large telescopes and ultra-stable spectrographs. Based on spectroscopic time series of a single Fe line, Kochukhov et al. (2002) claim the first possible detection of a pulsation mode in $\beta \mathrm{CrB}$ with a period of $11.5 \mathrm{~min}$. This result is questioned by Hatzes \& Mkrtichian (2004) and has also not been confirmed by Kurtz et al. (2007). However, the good agreement between the independent spectroscopic studies of Hatzes \& Mkrtichian (2004), Kurtz et al. (2007), and Kochukhov et al. (2008) confirm that $\beta \mathrm{CrB}$ is indeed an roAp star with a single known low-amplitude mode with period 16.2 min. The most robust result was found by Kurtz et al. (2007), who used $2 \mathrm{~h}$ of high-cadence time-series spectra obtained with VLT/UVES. They detected a single oscillation frequency at $1.031 \mathrm{mHz}(P=16.2 \mathrm{~min})$ with an amplitude of $23.5 \pm 2.4 \mathrm{~km} \mathrm{~s}^{-1}$ in the $\mathrm{H} \alpha$ line and a higher amplitude in the cesium lines. Unlike most roAp stars, variation was only observed in singly-ionized rare-earth elements, but not doubly ionized lines. The abundance analysis done by Kurtz et al. (2007) on their averaged spectrum confirmed earlier investigations by Ryabchikova et al. (2004). These analyses show that $\beta \mathrm{CrB}$ has an overabundance of rare-earth elements but only by about 1 dex, contrary to the 2-3 dex seen in most roAp stars.

\section{Observations and data reduction}

\subsection{VLT/NACO adaptive optics imaging}

We observed $\beta \mathrm{CrB}$ on 16 June 2008 using the Nasmyth Adaptive Optics System (NAOS; Rousset et al. 2003) of the Very Large Telescope (VLT), coupled to the CONICA infrared camera (Lenzen et al. 1998), abbreviated as NACO. We selected the smallest available pixel scale of $13.26 \pm 0.03 \mathrm{mas} / \mathrm{pix}$ (Masciadri et al. 2003), giving a field of view of $13.6^{\prime \prime} \times 13.6^{\prime \prime}$. This small scale resulted in good sampling of the point spread function (PSF). We employed the $J$ and $K$ filters of NACO, with respective bandpasses of $1.265 \pm 0.25 \mu \mathrm{m}$ and $2.18 \pm 0.35 \mu \mathrm{m}$, together with a neutral density filter (labelled "ND2_short", transmission $\approx 1.5 \%$ ) to avoid saturation of the detector. The transmission curves of these filters are available on the NACO instrument web page ${ }^{1}$.

We obtained 20 images in the $J$ band and 40 images in the $K$ band, each with an exposure time of $0.35 \mathrm{~s}$. This is the minimum full-frame integration time of CONICA. The $J$ band images were collected during $\approx 2 \mathrm{~min}$ around UT01:53:31, and the $K$ images in $\approx 4 \mathrm{~min}$ around UT01:58:20. During these observations the DIMM seeing at Paranal in the visible was good $\left(0.6-0.7^{\prime \prime}\right)$, resulting in a high Strehl ratio $(\approx 45-50 \%)$. The raw images were dark-subtracted, flat-fielded (using lamp flats), and corrected for bad pixels using IRAF ${ }^{2}$. On the NACO images,

\footnotetext{
${ }^{1}$ http://www.eso.org/instruments/naco/inst/filters. html

${ }^{2}$ IRAF is distributed by the NOAO, which is operated by the Association of Universities for Research in Astronomy, Inc., under cooperative agreement with the National Science Foundation.
}

we measured both the differential photometry and the differential astrometry of $\beta \mathrm{CrB} \mathrm{B}$ relatively to $\beta \mathrm{CrB} \mathrm{A}$ taken as the reference.

To measure the relative astrometry, we treated each image separately using the Yorick ${ }^{3}$ software package. We used a classical $\chi^{2}$ minimization to fit an extracted subimage of $\beta \mathrm{CrB} \mathrm{A}$ (with a size of $9 \times 9$ pixels) at the position of the fainter component $\mathrm{B}$. The interpolation of the shifted image of A was done in Fourier space. The adjusted parameters were the relative positions $d$ (RA) and $d(\mathrm{Dec})$, the flux ratio, and the background level, although we used only the relative separations for our astrometric analysis. To estimate the associated error bars, we used the bootstrapping technique described by Kervella et al. (2004a). This technique is also called "sampling with replacement" and consists of constructing a hypothetical, large population derived from the original measurements and estimate the statistical properties from this population. The technique allows us to compute meaningful confidence intervals without any assumption on the properties of the underlying population (e.g. a Gaussian distribution). We validated the adopted Fourier interpolation method by comparing the results with a simple Gaussian fit of the two PSF cores. The two methods yield exactly the same relative positions (within $150 \mu$ as), although the Gaussian fit has a slightly larger dispersion because of the mismatch of the slightly seeing-distorted PSF and the Gaussian function. We obtained the following vector separations along the RA and Dec directions of $\mathrm{B}$ relatively to $\mathrm{A}$, for the epoch of the observations (MJD 54633.08):

$$
\begin{aligned}
& d(\mathrm{RA})=177.84 \pm 0.09 \pm 0.40 \mathrm{mas} \\
& d(\mathrm{Dec})=-208.38 \pm 0.06 \pm 0.47 \text { mas }
\end{aligned}
$$

The two stated error bars are the statistical and systematic uncertainties, respectively. The latter includes the pixel scale uncertainty and the detector orientation uncertainty. These values correspond to a separation $r$ and position angle $\alpha$ (east of north) of:

$$
\begin{aligned}
& r=273.95 \pm 0.63 \text { mas } \\
& \alpha=139.52 \pm 0.13 \text { degrees. }
\end{aligned}
$$

These measurements were done on the $K$ images since they have the highest Strehl ratio. In the $J$ band, the Strehl ratio was lower and more unstable, resulting in a significantly variable background from A to B. Although its average value is not a concern, its slope tends to slightly shift the average apparent position of $B$, by -0.2 and -1.4 mas in RA and Dec, respectively (towards the lower left quadrant of Fig. 1). The average NACO image in the $K$ band is presented in Fig. 1, together with the orbit by Tokovinin (1984). Our astrometric measurement falls on the predicted orbit within only 7 mas. We find that the reference epoch of the orbital elements by North et al. (1998) appears to be late by approximately 300 days.

The photometry was obtained in two steps: (1) we obtained the combined photometry of the two stars; (2) we then measured the differential flux of B relative to A. We will discuss these steps in the following.

(1) The combined ADU count was computed from the NACO images by using a large aperture enclosing the full PSFs of the two stars. It was then converted to magnitude using the zero points obtained routinely by the observatory

\footnotetext{
${ }^{3}$ http://yorick. sourceforge.net/
} 


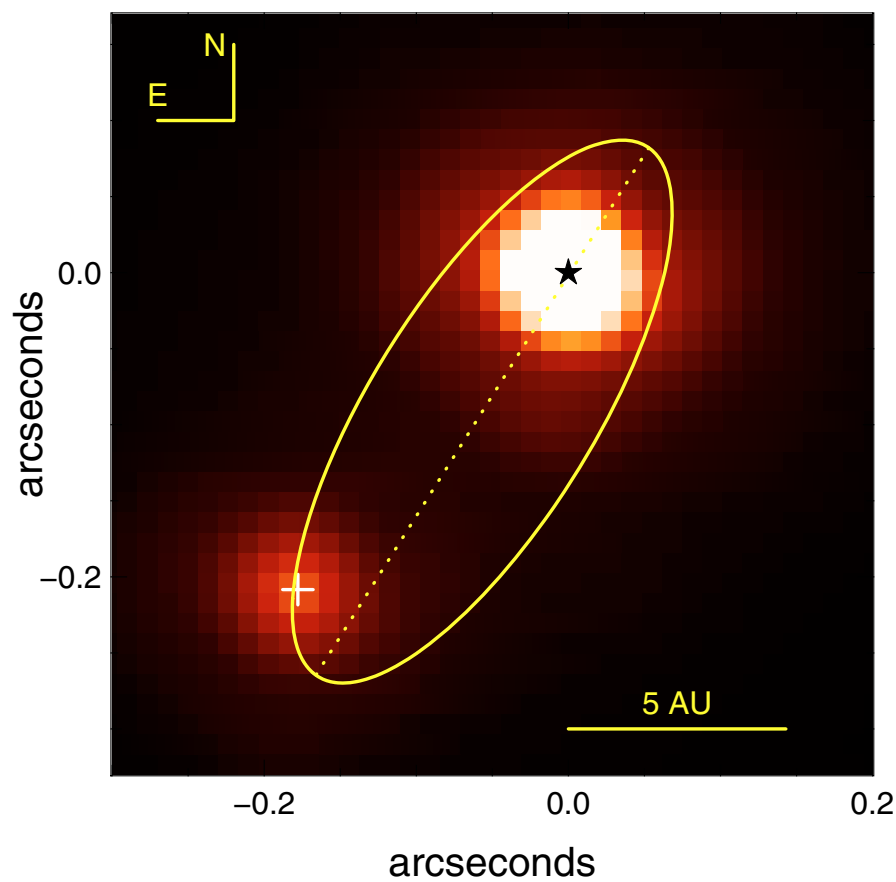

Fig. 1. Average NACO image of $\beta \mathrm{CrB}$ A and B in the $K$ band, together with the binary orbit from Tokovinin (1984). The positions of $\beta \mathrm{CrB} A$ and B measured on our NACO image are marked with " $\star$ " and "+" symbols, respectively. The scale of the two axes are in arc seconds, relative to $\beta \mathrm{CrB} \mathrm{A}$. The linear scale in $\mathrm{AU}$ is based on the Hipparcos parallax.

on the same night, $\mathrm{ZP}(J)=24.062 \pm 0.048$ and $\mathrm{ZP}(K)=$ $22.985 \pm 0.078$, and an attenuation of $4.81 \pm 0.03 \mathrm{mag}$ for the neutral density filter. These zero points have not been corrected for atmospheric absorption, but because they were obtained at low airmass $(\approx 1.15)$, we neglect the atmospheric absorption of $\approx 0.01 \mathrm{mag}$ in $J$ and $K$. We corrected the atmospheric absorption using the standard values by Nikolaev et al. (2000), namely $0.092 \mathrm{mag} / \mathrm{AM}$ (relative to unit airmass) for $J$ and $0.065 \mathrm{mag} / \mathrm{AM}$ for $K$, for our observation airmass of 1.71 . We obtain

$$
\begin{aligned}
& m_{J}(\mathrm{~A}+\mathrm{B})=3.28 \pm 0.07 \\
& m_{K}(\mathrm{~A}+\mathrm{B})=3.22 \pm 0.08
\end{aligned}
$$

(2) The differential photometry was obtained slightly differently, since the diffuse background of $\beta \mathrm{CrB}$ A tends to contaminate the flux of star B (but the reverse effect is negligible). We first computed aperture photometry of A on the average $J$ and $K$ images using very small aperture radii of 3 pixels in the $J$ band and 4 pixels in the $K$ band (contamination is lower in $K$ ). We calculated the median background value in concentric rings centered on A. This value was then subtracted from component B's flux. This allowed us to subtract the diffuse light from the PSF wings of A at the position of B. We checked that the residual background around B was negligible. We then integrated the flux of B on the ring-mediansubtracted image using the same aperture radius as for A. We obtain the following flux ratios of each star relative to the total of the two, i.e. $\rho(\star)=f(\star) / f(\mathrm{~A}+\mathrm{B})$ :

$$
\begin{aligned}
& \rho_{J}(\mathrm{~A})=0.7957 \pm 0.0084, \rho_{J}(\mathrm{~B})=0.2043 \pm 0.0021, \\
& \rho_{K}(\mathrm{~A})=0.7785 \pm 0.0002, \rho_{K}(\mathrm{~B})=0.2215 \pm 0.0002 .
\end{aligned}
$$

Table 1. Interferometric calibrators selected from Mérand et al. (2005).

\begin{tabular}{cccc}
\hline \hline HD & $\begin{array}{c}\text { Spectral } \\
\text { type }\end{array}$ & $\begin{array}{c}K \\
{[\mathrm{mag}]}\end{array}$ & $\begin{array}{c}\text { UD diameter } \\
\text { in } K \text { band [mas] }\end{array}$ \\
\hline 147266 & G8III & 3.8 & $0.785 \pm 0.011$ \\
145457 & K0III & 4.1 & $0.693 \pm 0.009$ \\
108123 & K0III & 3.7 & $0.929 \pm 0.012$ \\
\hline
\end{tabular}

The quoted uncertainties are statistical errors that do not include possible flat-fielding errors. From the combined magnitudes determined above, we obtain the individual magnitudes of $\beta \mathrm{CrB} \mathrm{A}$ and $\mathrm{B}$ :

$$
\begin{aligned}
& m_{J}(\mathrm{~A})=3.54 \pm 0.07, m_{J}(\mathrm{~B})=5.00 \pm 0.07 \\
& m_{K}(\mathrm{~A})=3.50 \pm 0.08, m_{K}(\mathrm{~B})=4.86 \pm 0.08
\end{aligned}
$$

The individual $J, K$ magnitudes have large uncertainties, but we stress that we only use the values of $\rho$ for the interpretation of our interferometric data (Sect. 2.2), and they are known with a much higher accuracy.

\subsection{CHARA/FLUOR interferometry}

Our interferometric observations of $\beta \mathrm{CrB}$ took place on 1718 May 2008 in the near infrared $K^{\prime}$ band $(1.9 \leq \lambda \leq 2.3 \mu \mathrm{m})$ at the CHARA Array (ten Brummelaar et al. 2005) using FLUOR (the Fiber Linked Unit for Optical Recombination; Coudé du Foresto et al. 2003). We used the FLUOR Data Reduction Software (DRS; Coudé Du Foresto et al. 1997; Kervella et al. 2004a; Mérand et al. 2006) to extract the squared instrumental visibility of the interference fringes. We used three different interferometric calibrators to calibrate the visibilities on sky. Their properties are listed in Table 1. We note that the angular diameters of the calibrator stars are comparable to or larger than the target star. Therefore they contribute significantly to the uncertainty of the angular diameter measurement. The corresponding systematic uncertainties were propagated into the final angular diameter uncertainties. The light from both stars of $\beta \mathrm{CrB}$ is injected simultaneously in the FLUOR fibers, since the acceptance angle is $0.8^{\prime \prime}$ on the sky. However, due to their on-sky separation of $\approx 0.3^{\prime \prime}$ (cf. Eq. (3)), two fringe packets are formed at different optical path differences.

For this reason, we have to correct our measured visibility for this effect. The monochromatic visibility of the binary is

$V=\rho_{K}(\mathrm{~A}) V(\mathrm{~A})+\rho_{K}(\mathrm{~B}) V(\mathrm{~B}) \mathrm{e}^{2 \mathrm{i} \pi \boldsymbol{B} \cdot \boldsymbol{\Gamma} / \lambda}$,

where $\rho_{K}(\mathrm{~A})$ and $\rho_{K}(\mathrm{~B})$ are the relative fluxes of $\mathrm{A}$ and $\mathrm{B}, V(\mathrm{~A})$ and $V(\mathrm{~B})$ are the individual visibilities, $\boldsymbol{B}$ the baseline vector, $\boldsymbol{\Gamma}$ the angular separation between $\mathrm{A}$ and $\mathrm{B}$, and $\lambda$ is the wavelength. Because we observed over a relatively broad wavelength range with FLUOR and since the binary is well-resolved by our baselines, the fringes appeared as two distinct fringe packets. Moreover, FLUOR has a limited window in terms of optical path difference, corresponding to a limited field of view. For $\beta \mathrm{CrB}$ $\mathrm{A}+\mathrm{B}$, the two fringe packets are not present in a single fringe scan. Hence, the squared visibility measured by FLUOR in the case of $\mathrm{A}$ is

$V^{2}=\rho_{K}(\mathrm{~A})^{2} V^{2}(\mathrm{~A})$.

In the case of the observations of $\mathrm{B}$, this multiplicative factor $\rho_{K}(\mathrm{~B})^{2}$ is small (because of the faintness of the star) and causes an amplification of the error bars on the true visibility $V^{2}(\mathrm{~B})$ for 
Table 2. Journal of observations.

\begin{tabular}{cccrcccc}
\hline \hline & $\begin{array}{c}\text { MJD } \\
\end{array}$ & $\begin{array}{c}B \\
(\mathrm{~m})\end{array}$ & $\begin{array}{r}\text { PA } \\
(\mathrm{deg})\end{array}$ & $\begin{array}{c}V^{2} \\
\text { observed }\end{array}$ & $\begin{array}{c}\text { \% } \\
V^{2}(\star) \% \\
\text { corrected }\end{array}$ \\
\hline $\mathrm{A}$ & 604.388 & 324.09 & 6.17 & 33.2 & \pm 1.6 & $54.7 \pm 2.7$ \\
$\mathrm{~A}$ & 604.408 & 323.61 & 1.40 & 31.5 & \pm 1.4 & $52.0 \pm 2.3$ \\
$\mathrm{~A}$ & 604.433 & 323.88 & -4.70 & $29.0 \pm 1.2$ & $47.8 \pm 2.0$ \\
$\mathrm{~A}$ & 604.488 & 327.21 & -17.40 & $34.1 \pm 3.6$ & $56.3 \pm 5.9$ \\
$\mathrm{~A}$ & 605.296 & 245.70 & -39.57 & $45.9 \pm 3.1$ & $75.8 \pm 5.1$ \\
$\mathrm{~A}$ & 605.326 & 248.92 & -45.62 & $44.7 \pm 2.2$ & $73.8 \pm 3.7$ \\
\hline B & 604.398 & 323.76 & 3.65 & $3.44 \pm 0.25$ & $70.0 \pm 5.2$ \\
B & 605.254 & 238.03 & -29.58 & $4.76 \pm 1.38$ & $97.1 \pm 28.0$ \\
\hline
\end{tabular}

Notes. $B$ is the projected length of the interferometric baseline, PA is the projection angle of the baseline, $V^{2}$ the observed squared visibility, and $V^{2}(\star)$ the squared visibility corrected for the presence of the other component (see Eq. (12)).

our second observation of this star. After reducing and calibrating the data with the DRS pipeline for each component, we corrected the visibilities for this effect and then derived the angular diameters using limb-darkened models from Claret (2000).

This leads to the following angular diameters:

$\theta_{\mathrm{LD}}(\mathrm{A})=0.699 \pm 0.017 \operatorname{mas}(2.4 \%)$,

$\theta_{\mathrm{LD}}(\mathrm{B})=0.515 \pm 0.054 \operatorname{mas}(10.5 \%)$.

The angular diameter of $\beta \mathrm{CrB} \mathrm{B}$ is significantly more uncertain than that of A, because of the very low apparent visibility (" $V^{2}$ observed" in Table 2) of its interference fringes "on top" of the incoherent flux from A. The large multiplicative factor $\rho_{K}(\mathrm{~B}) \approx$ 20 (Eq. (12)) also applies to the associated $V^{2}$ error bar. Only two measurements of $B$ could be derived from our data, and one of these points (MJD 54604.398) dominates the fit. In the case of A, our six data points are in fair agreement with the best-fit model with a reduced $\chi^{2}$ of 2.0 .

\subsection{Surface brightness-colour predictions}

We can compare the measured angular diameters of $\beta \mathrm{CrB} A$ and $\mathrm{B}$ with the predictions from the surface brightness-colour (hereafter SBC) relations calibrated by Kervella et al. (2004b) using their $(V, V-K)$ relation. The $K$ band magnitudes were obtained in Sect. 2.1. We derive the $V$ band magnitudes from the total magnitude of the system of $m_{V}(\mathrm{~A}+\mathrm{B})=3.67 \pm 0.05$ (Rufener 1988) and the magnitude difference $\Delta m=1.99 \pm 0.10$ measured by Horch et al. (2004) by speckle interferometry at $503 \mathrm{~nm}$. We adopted estimated uncertainties on $m_{V}$ and $\Delta m$ since they are not given explicitly in the references. This gives the component magnitudes in $V$,

$m_{V}(\mathrm{~A})=3.83 \pm 0.05, \quad m_{V}(\mathrm{~B})=5.82 \pm 0.10$,

and the predicted photospheric angular diameters:

$\theta_{\mathrm{LD}}(\mathrm{A})=0.696 \pm 0.028 \operatorname{mas}(4.0 \%)$,

$\theta_{\mathrm{LD}}(\mathrm{B})=0.415 \pm 0.017$ mas $(4.1 \%)$.

The predicted and measured angular diameters of $\beta \mathrm{CrB}$ A are therefore in excellent agreement, while they are satisfactory for $\mathrm{B}$ (difference of $1.7 \sigma$ ). We note that increasing the uncertainty on $m_{V}$ by a factor two does not significantly change the uncertainty of the angular diameters.

\subsection{Linear photospheric radii}

The original Hipparcos parallax of $\beta \mathrm{CrB}$ is $\pi=28.60 \pm$ 0.69 mas (Perryman \& ESA 1997), consistent with the new reduction by van Leeuwen (2007) of $\pi=29.17 \pm 0.76$ mas. However, as the new reduction is not corrected for binarity effects, we adopt the original Hipparcos parallax.

For $\beta \mathrm{CrB} \mathrm{A}$, the angular diameter measurement presented in Sect. 2.2 represents a significant improvement in accuracy, by a factor of 1.6, over the surface brightness-colour estimate of Sect. 2.3. For the B component, this is not the case, as the visibility measurement is made particularly difficult by the brighter A component. For the subsequent analysis presented in Sect. 3, we therefore choose to adopt our direct interferometric angular diameter measurement for $\beta \mathrm{CrB} \mathrm{A}$, while for $\mathrm{B}$ we use the $\mathrm{SBC}$ estimate computed from our $K$ band NACO photometry. This gives the following linear radii:

$$
\begin{aligned}
& R_{\text {interf }}(\mathrm{A})=2.63 \pm 0.09 R_{\odot}(3.4 \%) \\
& R_{\mathrm{SBC}}(\mathrm{B})=1.56 \pm 0.07 R_{\odot}(4.5 \%)
\end{aligned}
$$

\section{The effective temperatures and masses of $\beta \mathrm{CrB}$}

In the following we will determine the effective temperatures and luminosities of the components of $\beta \mathrm{CrB}$ using two methods. The first method (Sect. 3.1) relies on the bolometric correction (model-dependent), while the second method (Sect. 3.2) is only weakly model-dependent. We then compare the radius and $T_{\text {eff }}$ of the components with a grid of isochrones to determine their approximate age and evolutionary masses (Sect. 3.3).

\subsection{Luminosity and effective temperature from angular diameter $+B C+$ parallax}

We use the bolometric corrections $\left(B C_{V}\right)$ from Bessell et al. (1998). For the measured $V-K$ values of $0.36 \pm 0.09$ and $0.99 \pm 0.09$, for $\mathrm{A}$ and $\mathrm{B}$ we get $\mathrm{BC}_{V}(\mathrm{~A})=0.04, \mathrm{BC}_{V}(\mathrm{~B})=0.01$, $\mathrm{BC}_{K}(\mathrm{~A})=0.39$, and $\mathrm{BC}_{K}(\mathrm{~B})=1.01$ (with $\log g=4$ ). We assume the uncertainties on the $\mathrm{BCs}$ are $0.02 \mathrm{mag}$. We therefore obtain from the $V$ band photometry:

$m_{\text {bol }}(\mathrm{A})=3.87 \pm 0.05, \quad m_{\text {bol }}(\mathrm{B})=5.83 \pm 0.10$.

The same computation with the $K$ band magnitudes gives identical values within $0.01 \mathrm{mag}$. We thus obtain the following bolometric luminosities, assuming the Hipparcos parallax and $M_{\mathrm{bol}}=4.75$ for the Sun (recommendation by IAU 1999):

$L^{\mathrm{BC}}(\mathrm{A})=27.6 \pm 1.8 L_{\odot}, \quad L^{\mathrm{BC}}(\mathrm{B})=4.5 \pm 0.5 L_{\odot}$.

We can now use the radii determined in Sect. 2.4 to derive the effective temperatures of the two stars through $L / L_{\odot}=$ $\left(R / R_{\odot}\right)^{2}\left(T_{\text {eff }} / T_{\text {eff; } \odot}\right)^{4}$, where we use the solar value $T_{\text {eff; } \odot}=$ $5777 \mathrm{~K}(\operatorname{Cox} 2000)$ :

$T_{\mathrm{eff}}^{\mathrm{BC}}(\mathrm{A})=8160 \pm 200 \mathrm{~K}, \quad T_{\mathrm{eff}}^{\mathrm{BC}}(\mathrm{B})=6750 \pm 230 \mathrm{~K}$.

\subsection{Effective temperature from angular diameter + flux}

The above method for the determination of $T_{\text {eff }}$ has the caveat that it relies on the bolometric correction being valid for these stars. The BCs from Bessell et al. (1998) are calculated from atmospheric models and do not depend on the metallicity. As a check, we determine the $T_{\text {eff }}$ of the A component by a direct 


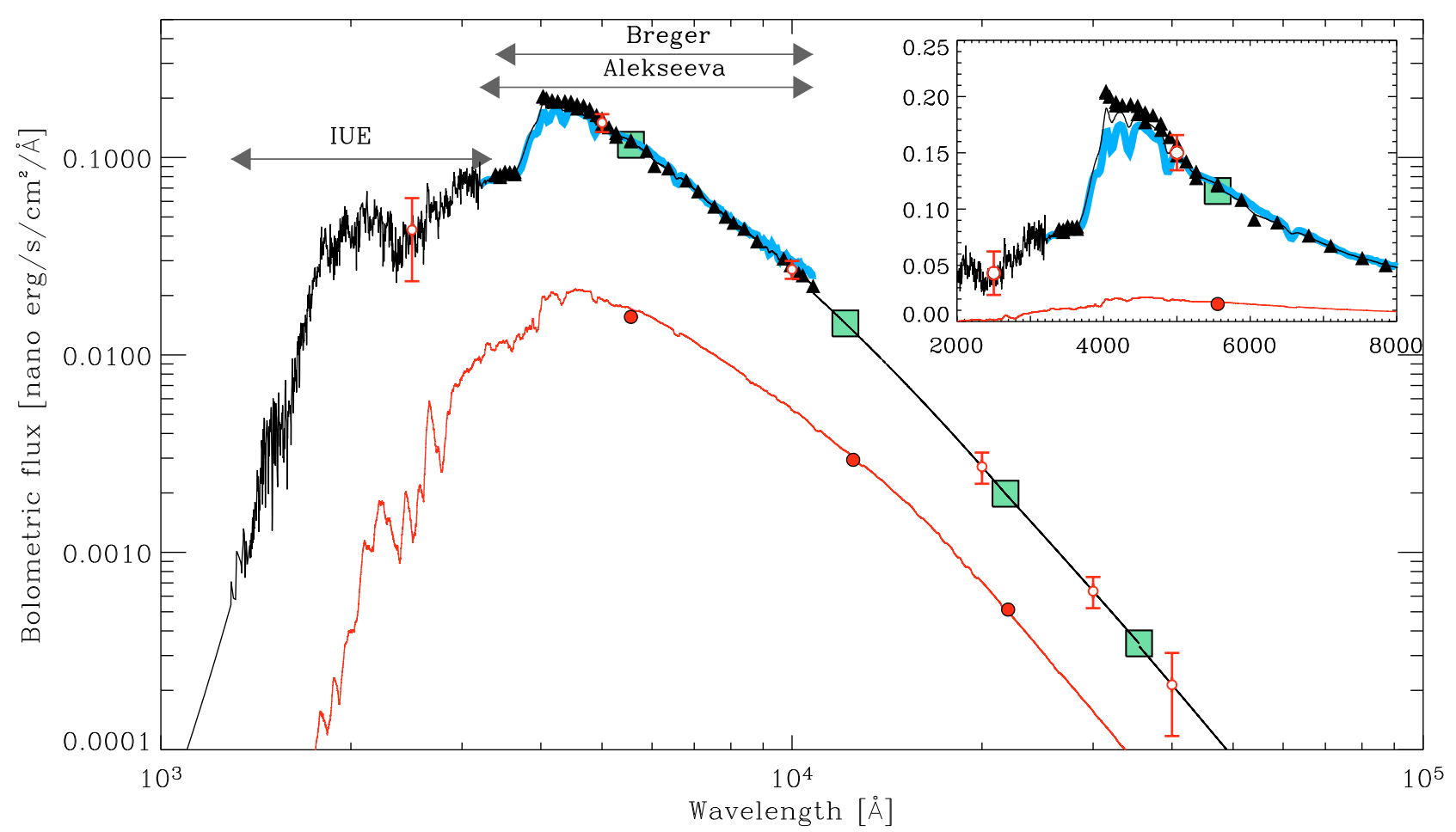

Fig. 2. The solid black line shows the flux distribution for $\beta \mathrm{CrB} \mathrm{A}+\mathrm{B}$, obtained by combining IUE satellite UV data, spectrophotometry from Breger (1976) (triangles) and Alekseeva et al. (1996) (thick curve), and broad band fluxes from Morel \& Magnenat (1978) (box symbols). The curve peaking at $\simeq 0.02 \times 10^{-9} \mathrm{erg} / \mathrm{s} / \mathrm{cm}^{2} / \AA$ is the ATLAS9 model spectrum fitted to the B component, scaled by fitting the $V$ and NACO $J, K$ magnitudes (filled circles). The open circles indicate the assumed 3-sigma error bars on the combined flux of the components at six different wavelengths. The inset shows the details from $2000-8000 \AA$ on a linear flux scale.

method, meaning it will only be weakly dependent on the assumed model atmosphere. This is done by calculating the integrated bolometric flux of $\mathrm{A}+\mathrm{B}$ and subtracting the flux from the $B$ component using an ATLAS9 model with the $T_{\text {eff }}$ determined above.

The bolometric flux of the combined system, $\beta \mathrm{CrB} \mathrm{A}+\mathrm{B}$, is obtained by combining data from the literature from the UV to the near IR as shown in Fig. 2. In the UV range we use five spectrograms from the Sky Survey Telescope (Jamar et al. 1976) obtained at the IUE "Newly Extracted Spectra" data archive ${ }^{4}$, covering the wavelength interval $1150 \AA<\lambda<3350 \AA$. We compute the weighted average of the spectrograms after removing some spurious data from 1150-1250 A. In the optical range we use spectrophotometry from Alekseeva et al. (1996) and Breger (1976), which cover the range $3200 \AA<\lambda<10800 \AA$. In addition, for the near-IR wavelengths we use the broadband magnitudes $V J K L$ from Morel \& Magnenat (1978). We interpolate the points between the broadband data and make a linear extrapolation at the end points (UV and IR ranges), although the contribution is negligible in practice. Finally, we calculate the weighted average flux, which is shown in Fig. 2. For the relative flux uncertainties we assume $15 \%$ in the UV, $6 \%$ in the optical, and $10 \%$ in the near IR. For the ranges where extrapolations were made, we double these errors. These adopted uncertainties are larger than the originally published values. We have adjusted them based on the disagreement between different sources of data in the same wavelength ranges, i.e. the spectrophotometric data from Breger (1976) and Alekseeva et al. (1996) in Fig. 2.

\footnotetext{
${ }^{4}$ http://sdc.laeff.inta.es/cgi-ines/IUEdbsMY/
}

Since $\beta \mathrm{CrB}$ is a binary system, extra care must be taken when computing the bolometric flux of the primary star. The binary has a maximum angular separation of $0.3^{\prime \prime}$ and all available flux data contain the combined light of the two components. Since our main interest is the A component, we have to estimate and subtract the flux of the B component. To accomplish this, we have fitted Kurucz models to the $m_{J}, m_{K}$ and $m_{V}$ magnitudes of the $B$ component, taking the statistical errors on the magnitudes into account. For the $m_{J}$ and $m_{K}$ magnitudes we adopt the measurements from NACO, while the $m_{V}$ magnitude is that derived in Eq. (15). The spectra for the Kurucz models are computed with the IDL routine kurget 1 (ATLAS9 models) and the corresponding database of models available in the IUE reduction and data analysis package IUEDAC ${ }^{5}$. We start by converting the $m_{V}$ magnitude of $\beta \mathrm{CrB}$ B into flux using relation (6) of Rufener \& Nicolet (1988) and use this result to calibrate the models. We then convert the Kurucz fluxes at the NACO $J$ and $K$ central wavelengths $(12650 \AA$ and $21800 \AA$ ) into magnitudes using $m=-2.5 \times \log _{10}\left(f / f_{0}\right)$. Here $m$ is the magnitude in a given filter, $f$ the flux at the central wavelength of that filter, and $f_{0}$ the standard zeroth-magnitude flux for the same filter. The values of $f_{0}$ are computed by integrating the flux of Vega through each of the $J$ and $K$ filters of the NACO instrument and assuming that Vega has zero magnitude in all bands ${ }^{6}$. We then generate 100 values for $m_{J}$ and $m_{K}$ of $\beta \mathrm{CrB}$ B by adding random fluctuations consistent with the uncertainties. For each set we determine the Kurucz model that fit each set of magnitudes

\footnotetext{
${ }^{5}$ http://archive.stsci.edu/iue/iuedac.html

${ }^{6}$ We note that Bohlin \& Gilliland (2004) recently found $V=0.026 \pm$ 0.008 for Vega.
} 
Table 3. Measured quantities and derived fundamental parameters.

\begin{tabular}{lcc}
\hline \hline Parameter & $\mathrm{A}$ & $\mathrm{B}$ \\
\hline$\pi$ [mas] & $28.60 \pm 0.69$ & Same as A \\
$\theta_{\mathrm{LD}}[\mathrm{mas}]$ & $0.699 \pm 0.017$ & $0.415 \pm 0.017$ \\
$f_{\text {bol }}\left[10^{-7} \mathrm{erg} / \mathrm{s} / \mathrm{cm}^{2}\right]$ & $6.6 \pm 0.5$ & $1.2 \pm 0.3$ \\
\hline$R / R_{\odot}$ & $2.63 \pm 0.09$ & $1.56 \pm 0.07$ \\
$L / L_{\odot}$ & $25.3 \pm 2.9$ & $4.5 \pm 0.5$ \\
$T_{\text {eff }}[\mathrm{K}]$ & $7980 \pm 180$ & $6750 \pm 230$ \\
Spectral type & $\mathrm{A} 5$ & $\mathrm{~F} 2$ \\
\hline$M / M_{\odot}$ & $2.09 \pm 0.15$ & $1.40 \pm 0.10$ \\
Age $[\mathrm{Gyr}]$ & $0.53 \pm 0.10$ & Same as A \\
\hline
\end{tabular}

best. The average integrated flux of the 100 fitted Kurucz models is $f_{B}=(1.2 \pm 0.3) \times 10^{-7} \mathrm{erg} / \mathrm{s} / \mathrm{cm}^{2}$. We adopt a rather large uncertainty $(25 \%)$ since we only have three broadband flux measurements of the B component.

The observed flux from the combined system is finally computed by integrating the black curve shown in Fig. 2, from which we obtain $f_{A}+f_{B}=(7.8 \pm 0.4) \times 10^{-7} \mathrm{erg} / \mathrm{s} / \mathrm{cm}^{2}$ and the bolometric flux of the primary component is thus $f_{A}=(6.6 \pm 0.5) \times 10^{-7}$ $\mathrm{erg} / \mathrm{s} / \mathrm{cm}^{-2}$. We can then obtain the effective temperature using the relation,

$\sigma T_{\mathrm{eff}}^{4}=\frac{4 f_{\mathrm{bol}}}{\theta_{\mathrm{LD}}^{2}}$,

where $f_{\mathrm{bol}}$ is the bolometric flux and $\theta_{\mathrm{LD}}$ is the limb-darkened angular diameter. Inserting the values for $f_{A}$ and the angular diameter from Sect. 2.2 we obtain an effective temperature of

$T_{\text {eff }}^{\theta}(\mathrm{A})=7980 \pm 180 \mathrm{~K}$.

Combining this with the radius we get the luminosity

$L^{\theta}(\mathrm{A})=25.3 \pm 2.9 L_{\odot}$.

These values agree with those in Sect. 3.1 where we used the (model dependent) bolometric correction. Since the calculation using Eq. 23 is nearly model-independent (limb-darkening coefficients depend on atmosphere models), we adopt these values as our final estimates of $T_{\text {eff }}$ and the luminosity. If we neglect the contribution to the flux from the B component, $T_{\text {eff }}^{\theta}$ (A) becomes $350 \mathrm{~K}$ higher.

Several determinations of $T_{\text {eff }}$ are found in the literature, and we mention a few here. Kochukhov \& Bagnulo (2006) used photometric indices to determine $T_{\text {eff }}$ and found $7430 \pm 200 \mathrm{~K}$, which is significantly lower than our value. Netopil et al. (2008) have determined the $T_{\text {eff }}$ of $\beta \mathrm{CrB}$ from three photometric systems (Strömgren, Geneva, and Johnson) and compared these with values in the literature. The mean value for the photometric indices is $7710 \pm 260 \mathrm{~K}$, and the mean of the literature values is $8340 \pm 360 \mathrm{~K}$. This is a typical example of the large scatter found for chemically peculiar A stars. However, since the rms scatter is large, the results summarized by Netopil et al. (2008) are in acceptable agreement with our new determination. It is worth stressing that our determination is the first that is not affected by photometric calibration errors or interstellar reddening and is only weakly dependent on the adopted limb darkening.

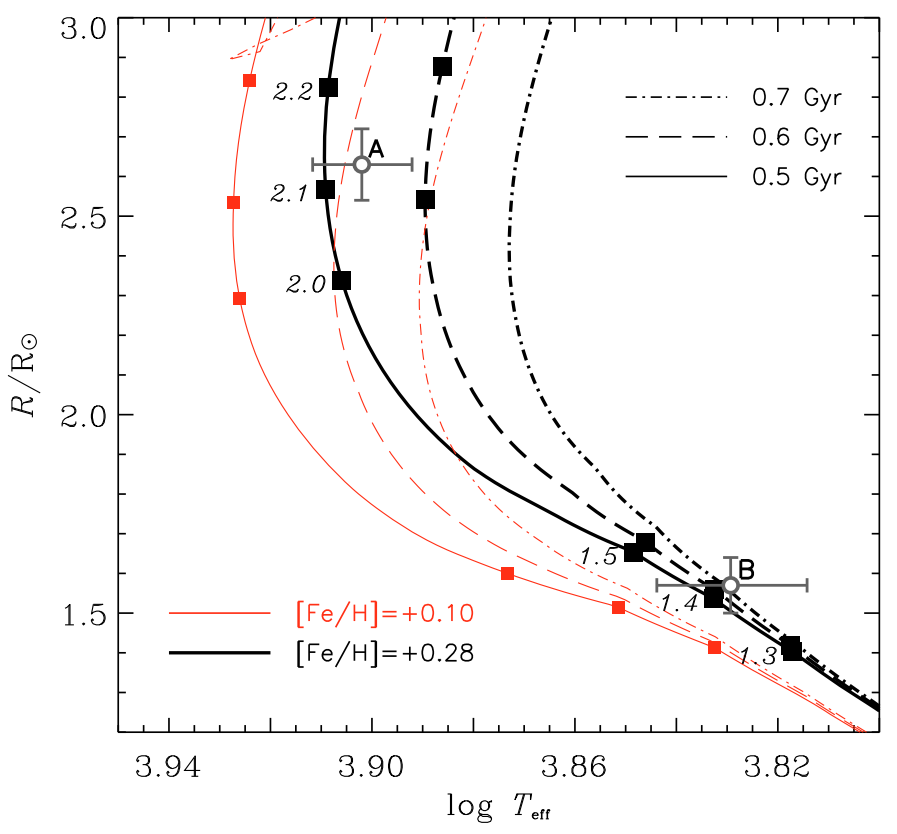

Fig. 3. Radius $-T_{\text {eff }}$ diagram showing the location of the two components of $\beta \mathrm{CrB}$. The thin lines are BASTI isochrones for $[\mathrm{Fe} / \mathrm{H}]=+0.10$ for ages $0.5,0.6$, and $0.7 \mathrm{Gyr}$. The thick lines are for the sames ages but for higher metallicity $[\mathrm{Fe} / \mathrm{H}]=+0.28$. On some of the isochrones the box symbols mark the masses at 1.3-1.5 $M_{\odot}$ and 2.0-2.2 $M_{\odot}$ in steps of $0.1 M_{\odot}$.

\subsection{The evolutionary status and mass of $\beta \mathrm{CrB}$}

To investigate the evolutionary status of the two components of $\beta \mathrm{CrB}$, we compare the observed $T_{\text {eff }}$ and radius with isochrones from the BASTI grid (Pietrinferni et al. 2004) as shown in Fig. 3. To transform the mass fraction of heavy elements $(Z)$ of the isochrones to spectroscopic $[\mathrm{Fe} / \mathrm{H}]$ values, we use the solar value $Z_{\odot}=0.0156$ (Caffau et al. 2009), i.e. $[\mathrm{Fe} / \mathrm{H}] \simeq$ $\log _{10}\left(Z_{\mathrm{BASTI}} / Z_{\odot}\right)$. We assume an uncertainty of \pm 0.002 on $Z_{\odot}$, which corresponds to \pm 0.05 dex on $[\mathrm{Fe} / \mathrm{H}]$.

In Fig. 3 we show two sets of isochrones with $[\mathrm{Fe} / \mathrm{H}]=$ +0.10 and +0.28 . The higher metallicity appears to be in better agreement with the location of the B component. Kurtz et al. (2007) find $[\mathrm{Fe}-\mathrm{II} / \mathrm{H}]=+0.41 \pm 0.22$ from 11 lines of singly ionized Fe. This is the metallicity in the photosphere, but we assume it represents the entire star. Since radial stratification of Fe is known to be present in roAp stars, our assumed metallicity is an approximation, but it seems to be supported by the agreement with the location of the stars in the radius $-T_{\text {eff }}$ diagram in Fig. 3. With this assumption, we determine the age to be $0.53 \pm 0.10 \mathrm{Gyr}$ and the masses of the components to be $M_{\mathrm{A}} / M_{\odot}=2.09 \pm 0.15$ and $M_{\mathrm{B}} / M_{\odot}=1.40 \pm 0.10$. These "evolutionary masses" are in good agreement with the dynamical masses determined by North et al. (1998): $M_{\mathrm{A}} / M_{\odot}=1.87 \pm 0.13$ and $M_{\mathrm{B}} / M_{\odot}=1.356 \pm 0.073$.

\section{Discussion and conclusion}

We have determined the effective temperature of the primary component of the binary $\beta \mathrm{CrB}$ using a technique that is only weakly model-dependent. We used interferometric data to measure the angular diameter and the fluxes were constrained using NACO $J, K$ measurements of each individual component in the binary. 
We determined the primary component of $\beta \mathrm{CrB}$ to have $T_{\text {eff }}=7980 \pm 180 \mathrm{~K}$. In comparison, literature values for the combined star span 7230 to $8700 \mathrm{~K}$ (considering $1-\sigma$ uncertainties). From a similar analysis of flux data and interferometric data on the roAp star $\alpha$ Cir, we found $T_{\text {eff }}=7420 \pm 170 \mathrm{~K}$ (Bruntt et al. 2008). For that star the literature values also span a wide range from 7470 to $8730 \mathrm{~K}$. It is interesting that, for $\beta \mathrm{CrB} \mathrm{A}$, our result is in the middle of the range of previous estimates while the estimate is at the low end of the range for $\alpha$ Cir. If we only compare $T_{\text {eff }}$ estimates from the same photometric system, Kochukhov \& Bagnulo (2006) find $7430 \pm 200 \mathrm{~K}$ for $\beta \mathrm{CrB}$ and $7670 \pm 200 \mathrm{~K}$ for $\alpha$ Cir. We must remember that the photometric index of $\beta \mathrm{CrB}$ includes both components and will always give a systematically low temperature. Taking this into account, the photometric values from Kochukhov \& Bagnulo (2006) seem to agree with our fundamental (i.e. model-independent) $T_{\text {eff }}$ values within about $\pm 300 \mathrm{~K}$. It will be necessary to make interferometric measurements of several more of the brightest Ap stars to be able to improve the $T_{\text {eff }}$ scale of these peculiar stars.

Accurate determinations of $T_{\text {eff }}$ have a strong impact on the asteroseismic modelling in future work. The roAp star $\alpha$ Cir was observed for 84 days with the $52 \mathrm{~mm}$ star tracker on the now defunct WIRE satellite. From the light curves Bruntt et al. (2009) detected five frequencies with two that had not been observed before. These two lie symmetrically around the well-known dominant mode at $2442 \mu \mathrm{Hz}$ to form a triplet. Bruntt et al. (2009) interpreted the equidistant separation as half the large separation. Combining this with the new $T_{\text {eff }}$, the properties of the star could be constrained based on preliminary theoretical modelling of the observed pulsation modes. To obtain similar results for $\beta \mathrm{CrB}$ would be worthwhile now that all ingredients for the modelling of the star are available, making it the second roAp star with well-established fundamental atmospheric parameters. This would require an ambitious asteroseismic campaign (Kurtz et al. 2007) using a network of telescopes with stable spectrographs like the Stellar Observations Network Group (SONG; Grundahl et al. 2008).

Our understanding of roAp stars would benefit from obtaining interferometric angular diameters of more targets. However, even with the most sensitive beam combiners currently available, only a handful are bright enough to yield a radius measurement to better than $2 \%$. Having now measured $\alpha$ Cir and $\beta \mathrm{CrB}$, we next propose to observe $\gamma$ Equ and 10 Aql.

Acknowledgements. The authors would like to thank all the CHARA Array and Mount Wilson Observatory daytime and nighttime staff for their support. The CHARA Array was constructed with funding from Georgia State University, the National Science Foundation, the W. M. Keck Foundation, and the David and Lucile Packard Foundation. The CHARA Array is operated by Georgia State University with support from the College of Arts and Sciences, from the Research Program Enhancement Fund administered by the Vice President for Research, and from the National Science Foundation under NSF Grant AST 0606958. S.T.R. acknowledges partial support from NASA grant NNH09AK731. M.S.C. acknowledges the support of the Portuguese MCTES and of the FSE, of the European Union, through the programme POPH. I.M.B. would like to acknowledge the support from the Fundação para a Ciência e Tecnologia (Portugal) through the grant SFRH/BD/41213/2007. L.L.K. is supported by the Lendület programme of the Hungarian Academy of Sciences. This research has made use of the Washington Double Star Catalog maintained at the US Naval Observatory. This work received the support of PHASE, the high angular resolution partnership between ONERA, Observatoire de Paris, CNRS, and University Denis Diderot Paris 7. This research took advantage of the SIMBAD and VIZIER databases at the CDS, Strasbourg (France), and NASA's Astrophysics Data System Bibliographic Services.

\section{References}

Alekseeva, G. A., Arkharov, A. A., Galkin, V. D. et al. 1996, Baltic Astron., 5, 603

Bessell, M. S., Castelli, F., \& Plez, B. 1998, A\&A, 333, 231

Bohlin, R. C., \& Gilliland, R. L. 2004, AJ, 127, 3508

Breger, M. 1976, ApJS, 32, 7

Bruntt, H., North, J. R., Cunha, M. et al. 2008, MNRAS, 386, 2039

Bruntt, H., Kurtz, D. W., Cunha, M. S. et al. 2009, MNRAS, 396, 1189

Caffau, E., Maiorca, E., Bonifacio, P. et al. 2009, A\&A, 498, 877

Campbell, W. W., \& Moore, J. H. 1907, Lick Observatory Bulletin, 4, 162

Claret, A. 2000, A\&A, 363, 1081

Coudé du Foresto, V., Borde, P. J., Mérand, A. et al. 2003, in SPIE Conf. Ser. 4838 , ed. W. A. Traub, 280

Coudé Du Foresto, V., Ridgway, S., \& Mariotti, J.-M. 1997, A\&AS, 121, 379

Cox, A. N. 2000, Allen's astrophysical quantities (Springer-Verlag)

Grundahl, F., Christensen-Dalsgaard, J., Arentoft, T. et al. 2008,

Communications in Asteroseismology, 157, 273

Hatzes, A. P., \& Mkrtichian, D. E. 2004, MNRAS, 351, 663

Horch, E. P., Meyer, R. D., \& van Altena, W. F. 2004, AJ, 127, 1727

Jamar, C., Macau-Hercot, D., Monfils, A. et al. 1976, Ultraviolet bright-star spectrophotometric catalogue. A compilation of absolute spectrophotometric data obtained with the Sky Survey Telescope (S2/68) on the European Astronomical Satellite TD-1

Kamper, K. W., McAlister, H. A., \& Hartkopf, W. I. 1990, AJ, 100, 239

Kervella, P., Ségransan, D., \& Coudé du Foresto, V. 2004a, A\&A, 425, 1161

Kervella, P., Thévenin, F., Di Folco, E., \& Ségransan, D. 2004b, A\&A, 426, 297

Kochukhov, O., \& Bagnulo, S. 2006, A\&A, 450, 763

Kochukhov, O., Landstreet, J. D., Ryabchikova, T., Weiss, W. W., \& Kupka, F. 2002, MNRAS, 337, L1

Kochukhov, O., Ryabchikova, T., Bagnulo, S., \& Lo Curto, G. 2008, Contributions of the Astronomical Observatory Skalnate Pleso, 38, 423

Kreidl, T. J. 1991, MNRAS, 248, 701

Kurtz, D. W. 1989, MNRAS, 238, 261

Kurtz, D. W., Elkin, V. G., \& Mathys, G. 2007, MNRAS, 380, 741

Lenzen, R., Hofmann, R., Bizenberger, P., \& Tusche, A. 1998, in SPIE Conf. Ser. 3354, ed. A. M. Fowler, 606

Masciadri, E., Brandner, W., Bouy, H. et al. 2003, A\&A, 411, 157

Mérand, A., Bordé, P., \& Coudé Du Foresto, V. 2005, A\&A, 433, 1155

Mérand, A., Coudé du Foresto, V., Kellerer, A. et al. 2006, in SPIE Conf. Ser., 6268

Morel, M., \& Magnenat, P. 1978, A\&AS, 34, 477

Muterspaugh, M. W., Lane, B. F., Kulkarni, S. R. et al. 2006, ApJ, 653, 1469

Netopil, M., Paunzen, E., Maitzen, H. M., North, P., \& Hubrig, S. 2008, A\&A, 491,545

Neubauer, F. J. 1944, ApJ, 99, 134

Nikolaev, S., Weinberg, M. D., Skrutskie, M. F. et al. 2000, AJ, 120, 3340

North, P., Carquillat, J.-M., Ginestet, N., Carrier, F., \& Udry, S. 1998, A\&AS, 130,223

Oetken, L., \& Orwert, R. 1984, Astron. Nachr., 305, 317

Perryman, M. A. C., \& ESA, eds. 1997, The HIPPARCOS and TYCHO catalogues. Astrometric and photometric star catalogues derived from the ESA HIPPARCOS Space Astrometry Mission, ESA Special Publication, 1200

Pietrinferni, A., Cassisi, S., Salaris, M., \& Castelli, F. 2004, ApJ, 612, 168

Renson, P., \& Manfroid, J. 2009, A\&A, 498, 961

Rousset, G., Lacombe, F., Puget, P. et al. 2003, in SPIE Conf. Ser. 4839, ed. P. L. Wizinowich, \& D. Bonaccini, 140

Rufener, F. 1988, Catalogue of stars measured in the Geneva Observatory photometric system : $4: 1988$ (Sauverny: Observatoire de Genève)

Rufener, F., \& Nicolet, B. 1988, A\&A, 206, 357

Ryabchikova, T., Nesvacil, N., Weiss, W. W., Kochukhov, O., \& Stütz, C. 2004, A\&A, 423, 705

Söderhjelm, S. 1999, A\&A, 341, 121

ten Brummelaar, T. A., McAlister, H. A., Ridgway, S. T. et al. 2005, ApJ, 628, 453

Tokovinin, A. A. 1984, Pisma Astronomicheskii Zh., 10, 293

Trilling, D. E., Stansberry, J. A., Stapelfeldt, K. R. et al. 2007, ApJ, 658, 1289

van Leeuwen, F., 2007, Astrophysics and Space Science Library, 250, Hipparcos, the New Reduction of the Raw Data

Weiss, W. W., \& Schneider, H. 1989, A\&A, 224, 101 